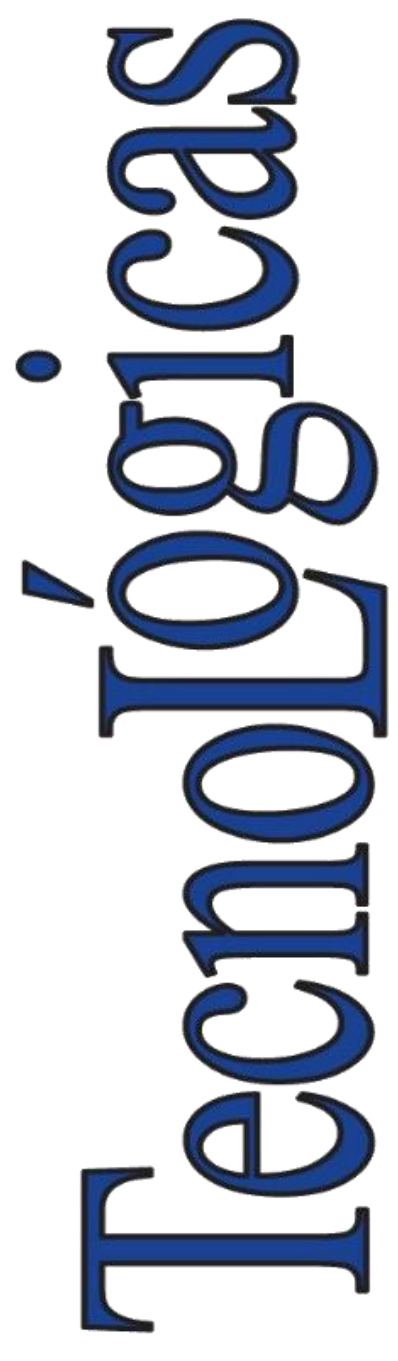

ISSN-p: 0123-7799 ISSN-e: 2256-5337

Vol. 24, nro. 51, e1830, 2021

Recibido: 9 diciembre 2020 Aceptado: 16 abril 2021 Disponible: 02 junio 2021

CInstituto Tecnológico Metropolitano Este trabajo está licenciado bajo una Licencia Internacional Creative Commons Atribución (CC BY-NC-SA)

\section{Aprovechamiento de residuos en la construcción de galpones como alternativa de sostenibilidad en el corregimiento El Prodigio, en San Luis, Antioquia-Colombia}

\author{
Using Wastes to Build Sheds as a Sustainable \\ Alternative in EI Prodigio (San Luis, Antioquia-Colombia) \\ (D) Hernán Darío Cañola1; \\ (iD Fidel Granda-Ramírez² \\ iD Kelly Leani Quintero-García ${ }^{3}$
}

${ }^{1}$ Institución Universitaria Colegio Mayor de Antioquia, MedellínColombia, hernan.canola@colmayor.edu.co

2 Institución Universitaria Colegio Mayor de Antioquia, MedellínColombia, carlos.granda@colmayor.edu.co ${ }^{3}$ Institución Universitaria Colegio Mayor de Antioquia, MedellínColombia, kelly.quintero@colmayor.edu.co

Cómo citar / How to cite

H. D. Cañola; F. Granda-Ramírez; K. L. Quintero-García, "Aprovechamiento de residuos en la construcción de galpones como alternativa de sostenibilidad en el corregimiento El prodigio, en San Luis, Antioquia-Colombia”, TecnoLógicas, vol. 24, nro. 51, e1830, 2021. https://doi.org/10.22430/22565337.1830 


\section{Resumen}

El sector de la construcción es un pilar fundamental para la economía y el desarrollo del país. En años recientes, este ha tenido un crecimiento a gran escala, pero también ha sido uno de los sectores donde se generan más residuos sólidos, tanto ordinarios como de construcción y demolición, los cuales son dispuestos en botaderos a cielo abierto o en escombreras, con una gestión deficiente, provocando diversos problemas a la comunidad cercana y al medio ambiente. El aprovechamiento de residuos sólidos aplicados a proyectos constructivos ha sido considerado como una alternativa de sostenibilidad ambiental; por lo anterior, en esta investigación se implementaron residuos de construcción y demolición, botellas de tereftalato de polietileno (PET, por sus siglas en inglés), tierra, guadua, residuos madereros, restos de alambre y envolturas plásticas en la construcción de galpones como alternativa de mejora en comunidades rurales afectadas por la violencia en Antioquia, específicamente en el corregimiento El Prodigio del municipio de San Luis. Como resultados se determinó que la implementación de materiales alternativos en la construcción de galpones permitió la integración social y la reducción del deterioro ambiental debido al uso de $1864 \mathrm{~kg}$ de materias primas residuales consideradas para la comunidad como desechos, y $279 \mathrm{~kg}$ de materiales no residuales, donde un elemento natural como la guadua ocupó el mayor valor porcentual con $95 \%$, y el $5 \%$ restante, correspondiente a materiales propios del sector de la construcción. Por lo tanto, la aplicación de la sostenibilidad dirigida al campo avícola promueve el uso de materiales alternativos en lugares donde es necesario su aprovechamiento como mejora ambiental y social; es por lo anterior que en esta investigación surge como objetivo general minimizar los residuos plásticos presentes en la parte urbana y rural de la zona de estudio.

\section{Palabras clave}

Construcción sostenible, integración social, reúso de materiales, botellas tereftalato de polietileno, residuos de construcción y demolición.

\section{Abstract}

The construction sector, fundamental for the economy and development of Colombia, has experienced an enormous growth in recent years. However, it is also one of the sectors that generate most solid wastes (ordinary, construction, and demolition wastes), which are disposed of in open or mismanaged dumps, causing various problems to nearby communities and the environment. The use of solid waste in construction projects has been considered an alternative for environmental sustainability. Consequently, in this study, construction and demolition waste, polyethylene terephthalate bottles, soil, guadua, wood waste, wire waste, and plastic wraps were used to build chicken coops in El Prodigio (San Luis, Antioquia, Colombia) as an alternative to improve the well-being of its rural communities affected by violence. As a result, it was determined that using alternative materials in the construction of chicken coops fostered social integration and reduced the environmental impact of $1864 \mathrm{~kg}$ of residual raw materials (usually considered waste) and $279 \mathrm{~kg}$ of non-residual materials. Guadua, a natural fiber, was the most commonly used material, as it composed $95 \%$ of the constructions described here; the remaining $5 \%$ was made of elements widely employed in the building industry. Applying sustainability principles in the poultry sector promotes the use of alternative materials in communities that need environmental and social improvements, such as the minimization of plastic waste in urban and rural areas.

\section{Keywords}

Sustainable construction, social integration, reused materials, polyethylene terephthalate bottles, construction and demolition waste. 


\section{INTRODUCCIÓN}

Ubicado al Noroeste de Colombia, Antioquia es uno de los 32 departamentos que conforman el país, caracterizándose por su riqueza agrícola, fauna, flora, fuentes hídricas, minerales, clima y ubicación geográfica. Adicionalmente, debido a su cercanía con los océanos Atlántico y Pacífico, y al estar atravesado por la cordillera de los Andes, es considerada una zona montañosa con gran biodiversidad [1]-[4]. Sin embargo, debido a sus riquezas y ubicación estratégica, el departamento ha sido afectado por conflictos armados desde las décadas del cuarenta y del cincuenta [5], lo que desencadenó que, ya en los años noventa, casi la totalidad de su territorio estuviera afectada por diferentes actores del conflicto como las FARC, el ELN, el EPL y diversos grupos de autodefensa. Los lugares más afectados correspondían a zonas rurales, en especial los pertenecientes a municipios del Suroriente antioqueño como San Francisco, Cocorná, San Luis, San Carlos, Granada, San Rafael, Guatapé, Alejandría, Concepción y aquellos con límites con los departamentos de Chocó y Bolívar y con la subregión del Bajo Cauca [6]. De esas zonas rurales ubicadas en aquellos municipios, el corregimiento El Prodigio, situado en San Luis, fue uno de los más afectados por las tomas guerrilleras, debido a que este territorio es un punto de interconexión con otros municipios como Puerto Boyacá (cuna del paramilitarismo), considerado como la ventana del Magdalena Medio [7]. El Prodigio fue dominado por las FARC en la década de los noventa, pero, a inicios del año 2000, fueron desalojadas por grupos paramilitares, en especial las Autodefensas Campesinas del Magdalena Medio (ACMM), otra rama del paramilitarismo, quienes se consolidaron en dicho lugar durante casi 20 años debido, en parte, al abandono al que fueron sometidos por parte del Estado, lo que con el pasar del tiempo conllevó a la estigmatización de su población [7].

Actualmente, no se presentan problemáticas de violencia en el municipio de San Luis ni en el corregimiento El Prodigio, esto como consecuencia de las intervenciones militares realizadas por parte del Estado colombiano; sin embargo, debido al inadecuado manejo de residuos en este municipio, el corregimiento El Prodigio presenta problemáticas ambientales. En San Luis, anualmente hay un incremento promedio del $11 \%$ en la generación de residuos sólidos, equivalentes a unas 5200 toneladas, donde aproximadamente el $8 \%$ es reutilizado [8]. Debido a la sobre producción de residuos de plástico y vidrio, y a la carencia de recolección por parte de empresas prestadoras del servicio de aseo, estos desechos terminan en zonas boscosas y afluentes hídricos de El Prodigio, como la quebrada El Prodigio o el Río Cocorná, el cual, posteriormente, desemboca en el río Magdalena, y este a su vez en el mar Caribe, aumentando así el deterioro de ecosistemas acuáticos [9]. Este tipo de situaciones que afectan al corregimiento, también son comunes en otras zonas rurales del mundo. No obstante esto, muchos investigadores han estudiado alternativas para minimizar estas problemáticas a través de la aplicación de la construcción sostenible con relación al aprovechamiento de residuos, como se muestra a continuación: como alternativa al deterioro ambiental debido a la sobreproducción de botellas de tereftalato de polietileno (PET) y a la carencia habitacional que se presenta en Colombia, [10] evaluaron las propiedades físico-mecánicas de estas botellas con rellenos de tierra y escombros como opción de mampuesto en la construcción de tanques y muros para viviendas alternativas. En su investigación demostraron que este tipo de materiales considerados como residuos presentan características de estabilidad para ser empleados en construcciones de bajo costo y posdesastre, además establecieron que las propiedades mecánicas como la resistencia a la compresión son similares a las de los ladrillos tradiciones aligerados con perforación horizontal. De igual manera, [11] analizaron la viabilidad del uso de botellas PET rellenas con diferentes tamaños de partículas de Residuos de Construcción y Demolición (RCD), esto como alternativa a los ladrillos de arcilla utilizados en tabiques, estableciendo que las botellas con residuos más finos presentan un mejor 
comportamiento mecánico en comparación a las botellas rellenas con partículas de tamaños superiores a $4.75 \mathrm{~mm}$, lo anterior debido a la reducción de espacios que presentan los residuos con partículas menores; además, determinaron que este material es una alternativa sostenible para el sector de la construcción debido al reúso de materiales y a las cero emisiones de carbono.

Por otro lado, [12] estudió el uso de residuos de polietileno de alta densidad como sustituto de la arena en la fabricación de concretos, esto como alternativa a la problemática ambiental que se presenta en el mundo debido a los residuos plásticos; en su investigación determinó que el uso de polietileno como agregado fino genera una reducción de la densidad de los concretos y esta es inversamente proporcional a la ductilidad; además, estableció que a partir de las adiciones de estos residuos plásticos en concretos, se logran materiales compuestos más livianos, mejorando la capacidad de trabajo. Del mismo modo, otros autores demostraron que la reutilización de residuos plásticos como PET en el sector de la construcción, logran ser una alternativa viable con relación a la mejora ambiental debido a su utilización en sistemas constructivos alternativos y no estructurales, correspondientes a morteros, bloques de concreto y bloques de tierra comprimida [13]-[16]. Debido a lo anterior, y a la problemática presente en El Prodigio, en esta investigación surge como objetivo general minimizar los residuos plásticos presentes en la parte urbana y rural de la zona de estudio (Figura 1), y como objetivos específicos la sensibilización ambiental comunitaria y la reutilización de residuos plásticos en la ejecución de proyectos constructivos alternativos relacionados con la avicultura como fuente de ingresos de este corregimiento; de ahí la viabilidad de aprovechar el PET en la construcción de galpones alternativos que permitan una mejora ambiental y, además, que sean de utilidad para esta comunidad rural.
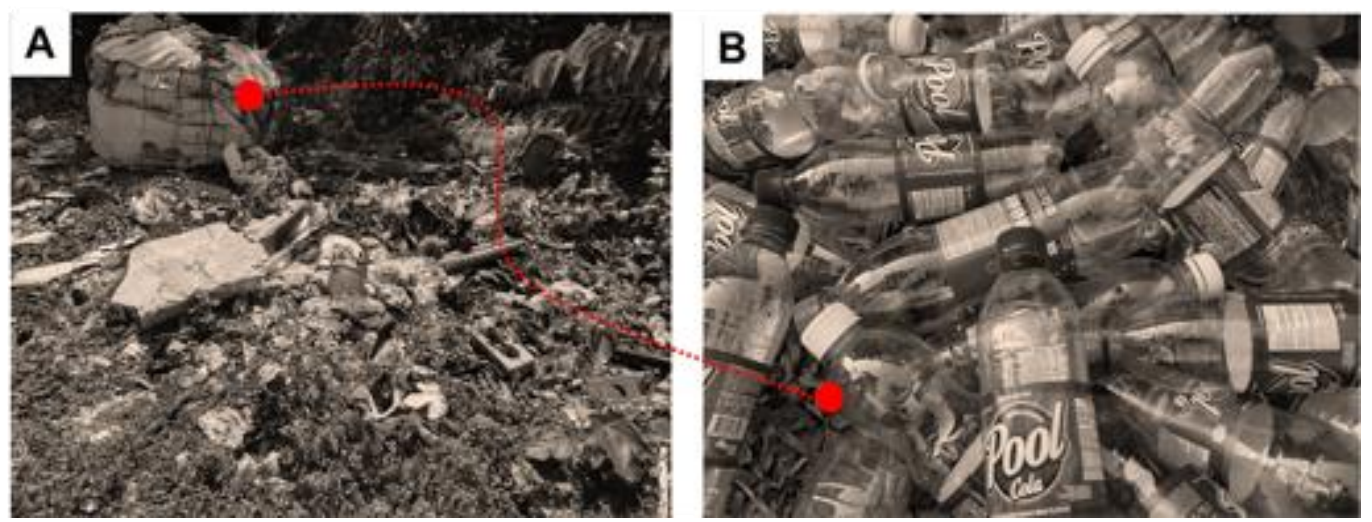

Figura 1. Residuos en zonas boscosas: A) Contenedor de plásticos y B) PET. Fuente: elaboración propia.

\section{METODOLOGíA}

Debido a la importancia de la problemática de estudio, en esta investigación de enfoque mixto se determinó, a través de la construcción sostenible, la importancia del aprovechamiento de residuos en la ejecución de elementos arquitectónicos alternativos como: muros, revoques, estructura vertical y horizontal, cubiertas y pisos, destinados a la ejecución de espacios correspondientes a la producción avícola. El desarrollo experimental de este proyecto se dividió en 5 etapas: i) Análisis de entorno, con la finalidad de determinar cuáles son los residuos y materiales más comunes y aprovechables en el corregimiento El Prodigio (considerados por la comunidad como residuos sólidos); ii) Análisis de materiales, con el propósito de establecer las caracterices físicas de los insumos a implementar (tierra, guadua, 
RCD, botellas PET y madera); iii) Propuesta arquitectónica, la cual permitirá idealizar el proyecto mediante los diseños correspondientes a las diferentes vistas (fachada y 3D), iv) Construcción y puesta en funcionamiento, esto de forma conjunta con la comunidad y mediante capacitaciones técnicas previas; y v) Se procedió a verificar, mediante procesos de inspección ocular, el comportamiento de los sistemas constructivos frente a los agentes externos, lo anterior posterior a nueve meses de su construcción.

\section{RESULTADOS}

\subsection{Etapa 1: análisis de entorno}

El Prodigio se encuentra localizado en el municipio de San Luis al oriente de Antioquia, en Colombia (Figura 2), se caracteriza por sus ecosistemas kársticos y riqueza en mármol, de ahí que este material pétreo se encuentre distribuido en toda la superficie de estudio. Para realizar esta etapa preliminar se efectuó una entrevista a 20 estudiantes de la Tecnología en Gestión Ambiental de la Institución Universitaria Colegio Mayor de Antioquia sobre las problemáticas ambientales y las necesidades presentes en el corregimiento El Prodigio. Todos los encuestados, pertenecientes a la comunidad de estudio, estaban conformados en su mayoría (90\%) por madres cabezas de familia, con un rango de edades entre los 20 años y los 45 años.

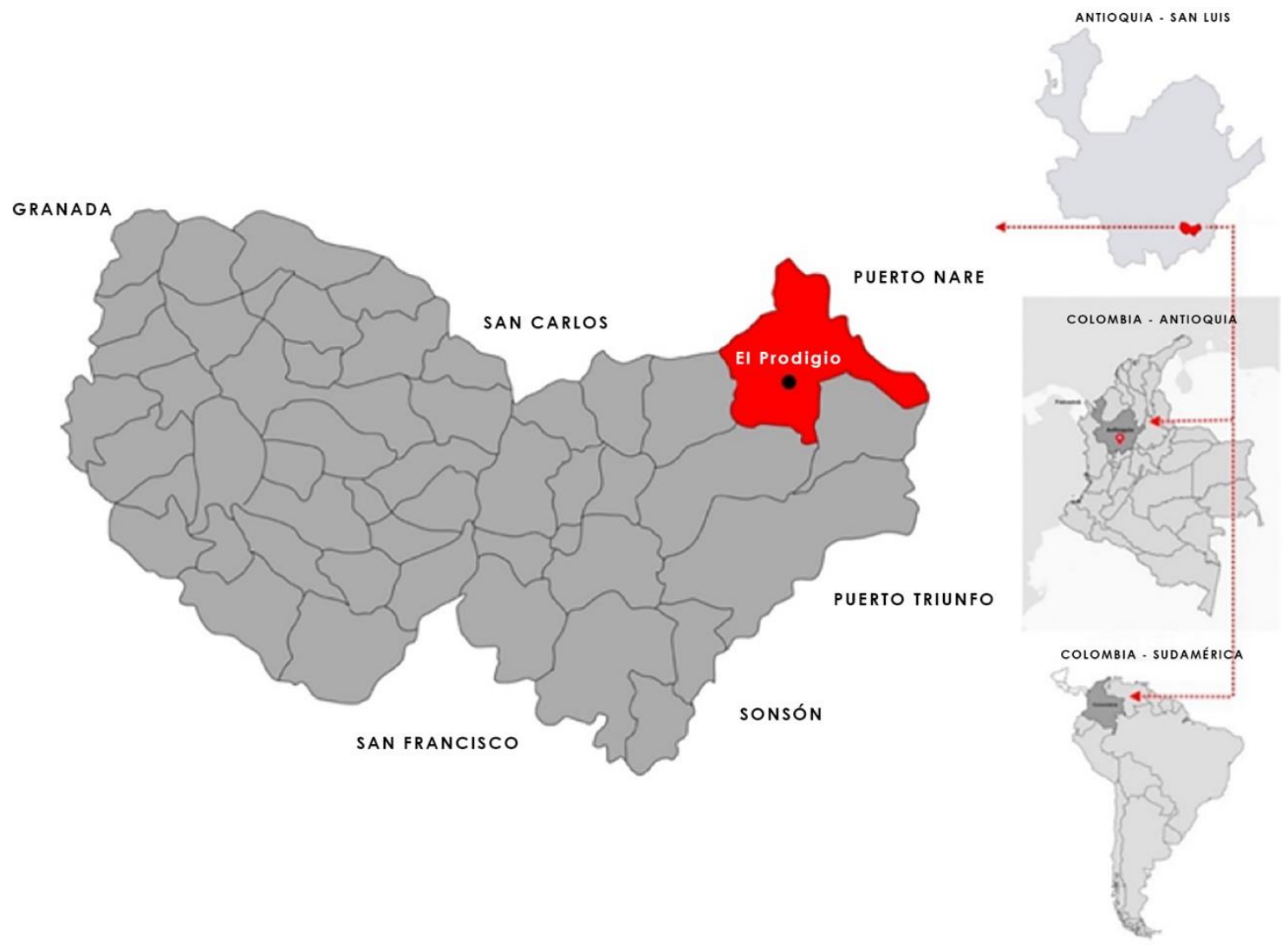

Figura 2. Geolocalización del corregimiento El Prodigio. Fuente: elaboración propia. 
Una vez analizadas las problemáticas ambientales relacionadas con la sobreproducción de residuos, se procedió a hacer la verificación mediante la realización de una inspección visual de forma conjunta con los estudiantes; lo anterior como método de recolección de información, con la cual se determinó que las zonas urbanas y boscosas carecían de acopios para la disposición de residuos sólidos; de ahí que la presencia de botellas PET y envolturas plásticas fueran un problema ambiental para la comunidad, situación que fue considerada punto de partida para el aprovechamiento de estos residuos mediante la aplicación de la construcción sostenible. Además de los desechos plásticos, se pudo establecer que la presencia de recursos como guadua, retales de madera, tierra y RCD eran de fácil disponibilidad. Este tipo de materiales y residuos han sido implementados a través de la arquitectura en la construcción de edificaciones como alternativa de sostenibilidad ambiental [17]-[22]. Debido a los anteriores fundamentos teóricos que soportan el uso de estos recursos en la construcción de edificaciones, se podría decir que su uso puede estar direccionado a la construcción de prototipos arquitectónicos de aprovechamiento avícola (galpones y gallineros) como medio de sostenibilidad para comunidades rurales con problemáticas ambientales, sociales y económicas.

\subsection{Etapa 2: análisis de materiales}

Una vez realizado el análisis de entorno e identificadas las problemáticas respecto a la presencia de residuos sólidos (botellas PET), y estudiada la disponibilidad de otros recursos alternativos (tierra, madera, guadua y RCD), se procedió con la caracterización física de estos materiales. Las densidades fueron determinadas mediante correlaciones de masas y volúmenes, garantizando siempre que los recursos analizados estuvieran secos y con una humedad de equilibrio del $10 \%$ al $13 \%$ en materiales higroscópicos. Los resultados obtenidos se muestran en la Tabla 1.

Tabla 1. Densidad de materias primas. Fuente: elaboración propia.

\begin{tabular}{cc}
\hline Material & Densidad $\left(\mathrm{kg} / \mathrm{m}^{3}\right)$ \\
\hline Tierra & 1650 \\
Guadua & 635 \\
Cedro & 450 \\
PET & 1350 \\
RCD & 1850 \\
\hline
\end{tabular}

Para la caracterización, inicialmente se analizó la tierra proveniente de zonas boscosas de El Prodigio mediante pruebas organolépticas de campo [23], [24], [25], la cual fue extraída a una profundidad aproximada de $0.5 \mathrm{~m}$; posteriormente, en un laboratorio de manera controlada se realizaron pruebas de granulometría bajo parámetros de la norma [26] y medición de $\mathrm{pH}$ mediante un medidor multiparámetros LAQUAact PC110K, con el cual se obtuvo como resultado un valor de 7.88, correspondiente a un $\mathrm{pH}$ básico. El registro fotográfico de algunas pruebas realizadas se muestra en las Figura 3. Las pruebas de granulometría establecieron que la tierra analizada corresponde a un suelo fino-granular medio con un módulo de finura de 2.49 [27] (Figura 4), cuyo uso fue destinado a la fabricación de revoques como componente constructivo de los galpones. 

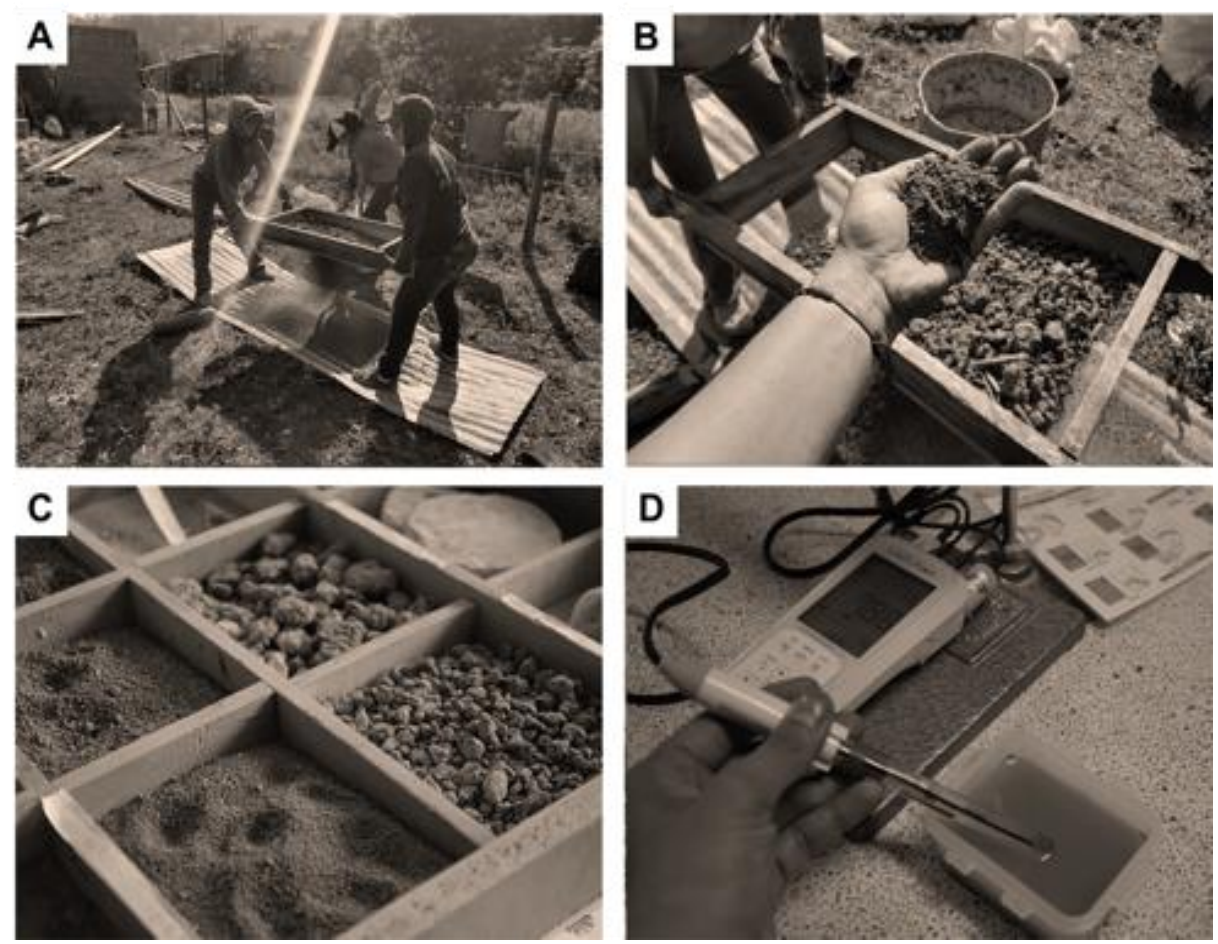

Figura 3. Análisis de suelos: A) Tamizado, B) Prueba de olor, C) Separación granulométrica y D) Medición de pH. Fuente: elaboración propia.

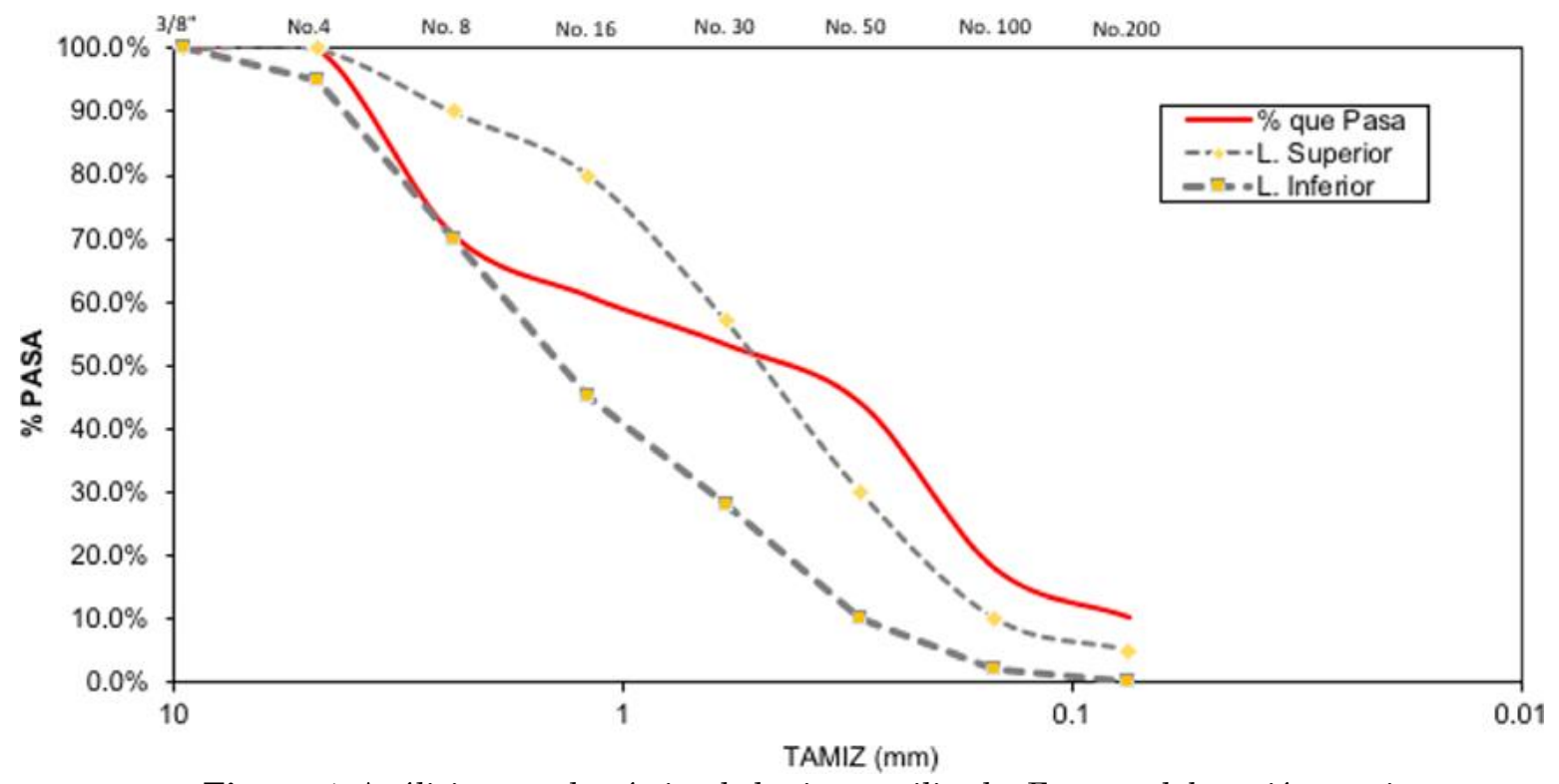

Figura 4. Análisis granulométrico de la tierra utilizada. Fuente: elaboración propia.

Posterior al análisis de la tierra, se procedió con la caracterización de residuos de madera y de guadua presentes en las zonas boscosas y urbanas de El Prodigio; para este estudio se tomaron cuatro muestras de cada uno de los recursos forestales, las cuales fueron sometidas a un proceso de secado en un laboratorio a temperatura constante de $(100 \pm 3){ }^{\circ} \mathrm{C}$ durante 24 horas [28]; luego se analizaron sus densidades (Tabla 1).

Se efectúa análisis organoléptico de las muestras de madera (Tabla 2) con la finalidad de determinar su tipología a través del uso de fuentes bibliográficas para la identificación de 
recursos madereros [29]; las muestras analizadas corresponden a cedro y la guadua Angustifolia Kunth que crece en la región de análisis donde es usada comúnmente en la realización de acabados, elementos decorativos y muebles. La Figura 5 muestra algunas de las pruebas realizadas.

Tabla 2. Pruebas organolépticas del Cedro. Fuente: elaboración propia.

\begin{tabular}{cc}
\hline Prueba & Densidad $\left(\mathrm{kg} / \mathrm{m}^{3}\right)$ \\
\hline Olor & Agradable, suave \\
Brillo & Poco brillo, opaco \\
Color & Rojizo \\
Textura & Superficie lisa y suave \\
Sabor & Amargo \\
\hline
\end{tabular}
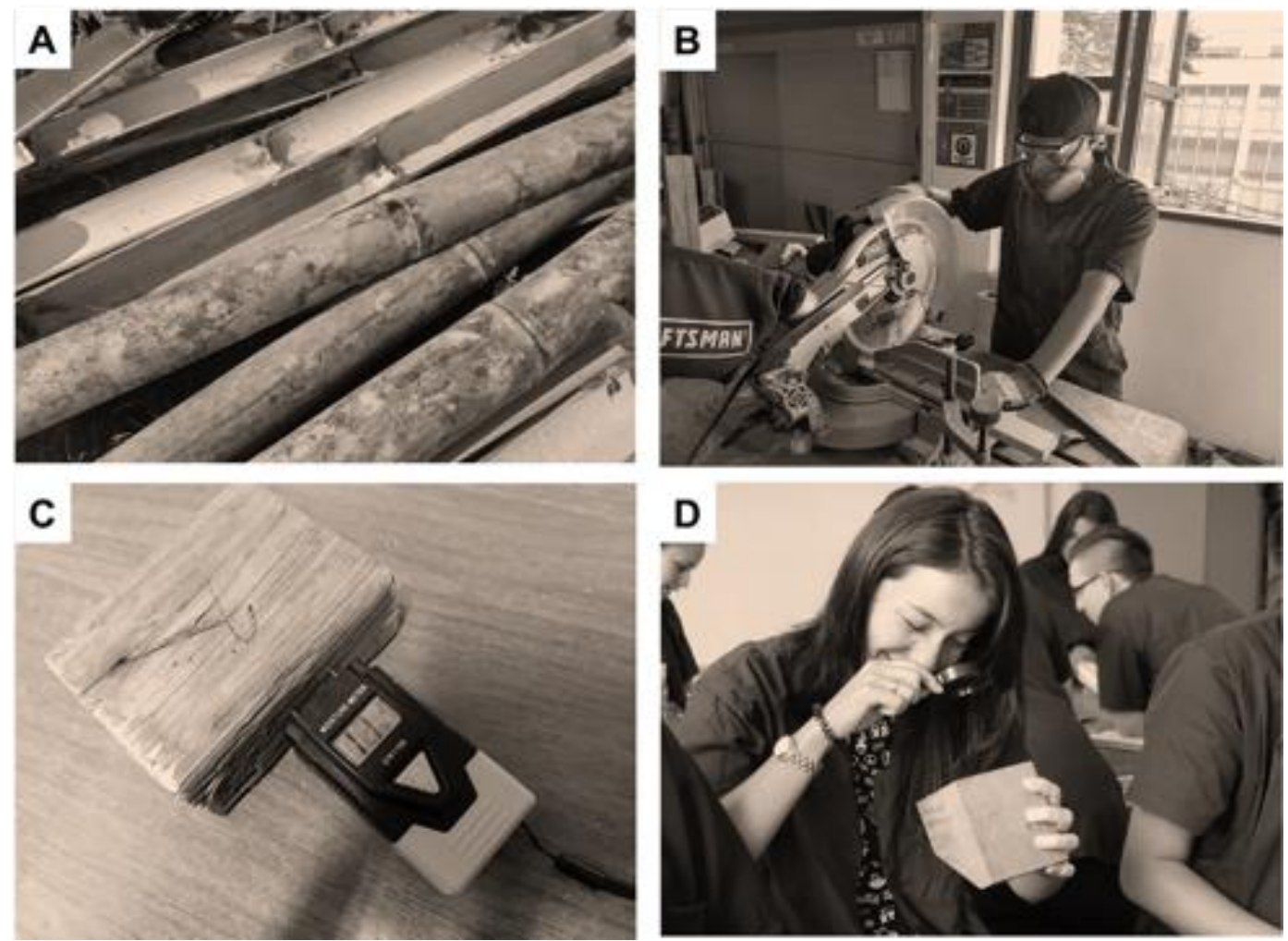

Figura 5. Análisis de guadua y madera: A) Guadua, B) Corte de madera, C) Medición humedad de equilibrio y D) Inspección ocular. Fuente: elaboración propia.

Por último, se procede con la caracterización de los RCD y las botellas PET; los residuos de demolición y construcción en su gran mayoría corresponden a restos de concreto, los cuales fueron triturados, tamizados y sometidos a un proceso de análisis granulométrico bajo parámetros de la norma [26]; además, se determinó la presencia de material orgánico mediante el ensayo de colorimetría establecido por la norma [30]. Según los ensayos realizados se establece que el RCD de concreto corresponde a un material fino-granular muy grueso con un módulo de finura de 5.76 [27] y la presencia de material orgánico es mínima según la escala colorimétrica de Gardner, debido a la coloración amarilla clara situada por debajo del límite colorimétrico correspondiente al color marrón. Con relación a la caracterización de las botellas PET que representan la problemática ambiental en el corregimiento El Prodigio, se establece que este material presenta una coloración translucida, 
buena resistencia química frente agentes externos, durabilidad, impermeabilidad y estabilidad física; de ahí que este material sea utilizado ampliamente a en el mundo como alternativa constructiva [10], [13], [31]-[33]; alguna de las propiedades fisicomecánicas del PET se muestran en la Tabla 3.

Tabla 3. Propiedades del PET. Fuente: elaboración propia.

\begin{tabular}{cccc}
\hline Tipología & Prueba & Valor & Referencia \\
\hline \multirow{3}{*}{ Mecánica } & Módulo de elasticidad (GPa) & $2-4$ & {$[34]$} \\
& Resistencia a la tracción (GPa) & 80 & {$[34]$} \\
& Tensión por elongación (\%) & $50-300$ & {$[35]$} \\
\multirow{2}{*}{ Físicas } & Absorción de agua (\%) & $<0.7$ & {$[34]$} \\
& Compresibilidad (TPa) & 3.86 & {$[36]$} \\
\hline
\end{tabular}

\subsection{Etapa 3: propuesta arquitectónica}

Una vez estudiados los materiales para la construcción de los galpones, se procedió con la ejecución de los diseños arquitectónicos (Figura 6). Para la realización de estos, se analizó previamente la función que desempeñarían los materiales dentro del proyecto, con la finalidad de garantizar estabilidad, funcionalidad y estética (Tabla 4).
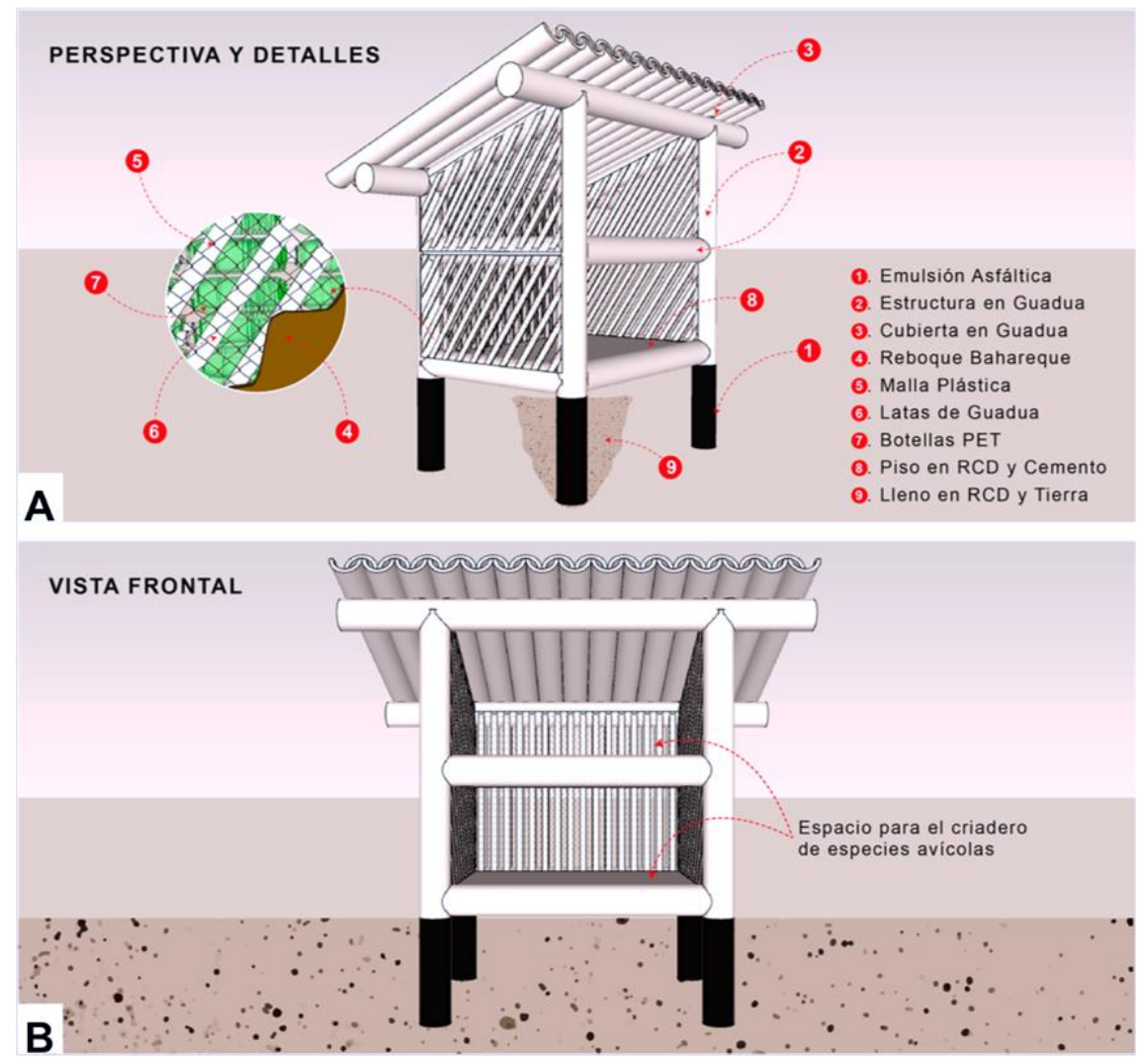

Figura 6. Diseños: A) Isométrico y componentes constructivos y B) Vista frontal. Fuente: elaboración propia. 
Tabla 4. Caracterización de materiales por tipología y uso. Fuente: elaboración propia.

\begin{tabular}{cccc}
\hline $\begin{array}{c}\text { Tipología según } \\
\text { su origen }\end{array}$ & Material & Elemento constructivo & Función \\
\hline Residual & Tierra & Revoque (Bahareque) & Estética \\
Residual & Madera & Viguetas & Estabilidad \\
Residual & Botellas PET & Muros, lleno y aislamiento & $\begin{array}{c}\text { Funcionalidad y } \\
\text { estabilidad }\end{array}$ \\
No residual & Emulsión asfáltica Sika ${ }^{\circledR}$ & Impermeabilizante & Funcionalidad \\
Residual & Boñiga & Revoque (Bahareque) & Funcionalidad \\
No residual & Cemento & Piso y estabilizante & Funcionalidad \\
Residual & Envolturas plásticas & Relleno de Botellas PET & Funcionalidad \\
No residual & Tornillos & Conectores & Estabilidad \\
No residual & Malla plástica Proplantas S.A. & Malla de amarre Revoque & Estabilidad \\
Residual & Restos de alambre & (Bahareque) & Estabilidad \\
No residual & Guadua & Amarres & Estabilidad y \\
Residual & RCD & Columnas, compuertas, muros & Funcionalidad \\
\end{tabular}

Los planos arquitectónicos fueron desarrollados conforme a las necesidades presentadas por la comunidad y su construcción fue determinada como una alternativa de sostenibilidad, mejora socioambiental y económica de las familias más desfavorecidas. Las dimensiones del galpón para un área de $4.8 \mathrm{~m}^{2}$, con una distribución de $2.4 \mathrm{~m}^{2}$ en la parte inferior y de $2.4 \mathrm{~m}^{2}$ en la parte superior, correspondieron a: altura de la fachada frontal: $2.5 \mathrm{~m}$, altura de la fachada posterior: $1.5 \mathrm{~m}$, ancho entre ejes de columna: $1.2 \mathrm{~m}$, largo entre ejes de columnas: $2 \mathrm{~m}$.

\subsection{Etapa 4: construcción y puesta en funcionamiento}

Para la tangibilidad de los diseños arquitectónicos, inicialmente se procedió con la cuantificación de materias primas y elementos constructivos (Tabla 5), esto mediante el despiece de los planos arquitectónicos y la correlación entre volúmenes y densidades de los materiales (Tabla 1) necesarios para la construcción de un galpón.

Por otro lado, en la Tabla 6 se muestra la inversión en materiales no residuales realizada para la construcción del galpón, excluyendo la guadua que era un material abundante en el sector.

Como actividades previas a la realización del galpón, inicialmente se designó el lugar de construcción y al mismo tiempo se realizó el proceso de recolección de materiales residuales como botellas PET y envolturas plásticas para su llenado; la distribución por cantidades porcentuales de cada uno de los materiales utilizados se muestra en la Figura 7.

Para la construcción del galpón intervinieron madres cabezas de familia con sus respectivos hijos y familiares (Figura 8); lo anterior como un proceso de sensibilización y educación ambiental en el corregimiento El Prodigio. 
Tabla 5. Cuantificación de materiales. Fuente: elaboración propia.

\begin{tabular}{|c|c|c|c|}
\hline Material & Elemento constructivo & Masa (kg) & Cantidad \\
\hline RCD & Llenos & 1665 & $0.9 \mathrm{~m}^{3}$ \\
\hline Madera & Viguetas inferiores & 11 & $2.2 \mathrm{~m}$ \\
\hline Guadua & Viguetas superiores & 43 & $17.3 \mathrm{~m}$ \\
\hline Guadua & Columnas & 25 & $10 \mathrm{~m}$ \\
\hline Guadua & Cubierta & 150 & $7.7 \mathrm{~m}^{2}$ \\
\hline Guadua & Piso intermedio & 14 & $2 \mathrm{~m}^{2}$ \\
\hline Guadua & Muros (Esterilla) & 31 & $6.2 \mathrm{~m}^{2}$ \\
\hline Emulsión asfáltica Sika® & Impermeabilizante & 0.6 & $0.8 \mathrm{~m}^{2}$ \\
\hline Boñiga & Revoque (Bahareque) & 30 & 1 saco \\
\hline Cemento & Piso y estabilizante & 15 & 0.3 bulto \\
\hline Envolturas & Relleno de Botellas & 44 & 1 saco \\
\hline Tornillos & Conectores & 0.5 & 1 caja \\
\hline Malla plástica Proplantas S.A. & (Bahareque) & 0.09 & $6.2 \mathrm{~m}^{2}$ \\
\hline Restos de alambre & Amarres & 2 & $2 \mathrm{~kg}$ \\
\hline Tierra & Revoque (Bahareque) & 103 & $3.1 \mathrm{~m}^{2}$ \\
\hline Botellas PET & Cerramiento, lleno y aislamiento & 9 & 434 unidades \\
\hline
\end{tabular}

Tabla 6. Costo de materiales no residuales. Fuente: elaboración propia.

\begin{tabular}{ccccc}
\hline Material & Elemento constructivo & Cantidad & $\begin{array}{c}\text { Valor } \\
\text { unitario } \$\end{array}$ & $\begin{array}{c}\text { Valor } \\
\text { total } \$\end{array}$ \\
\hline Emulsión asfáltica Sika ${ }^{\circledR}$ & Impermeabilizante & $0.8 \mathrm{~m}^{2}$ & 5000 & 4000 \\
Cemento & Piso y estabilizante & $15 \mathrm{~kg}$ & 1500 & 22500 \\
Tornillos & Conectores & $0.5 \mathrm{~kg}$ & 10000 & 5000 \\
Malla plástica Proplantas S.A. & (Bahareque) & $6.2 \mathrm{~m}^{2}$ & 12000 & 74400 \\
\hline & \multicolumn{3}{c}{ Valor total invertido en materiales $\$$} & 105900 \\
\hline
\end{tabular}

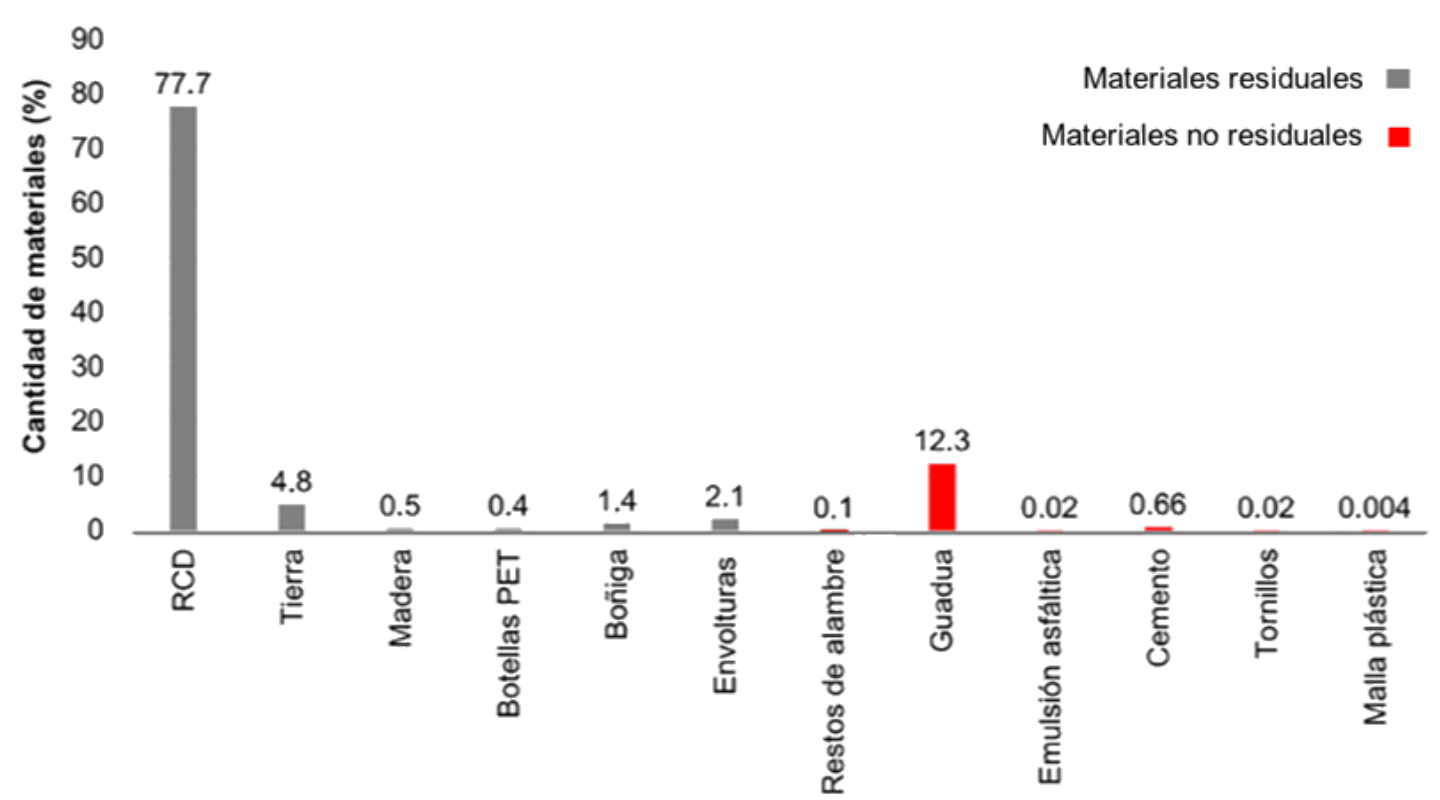

Figura 7. Cantidades porcentuales de materiales residuales y no residuales Fuente: elaboración propia. 

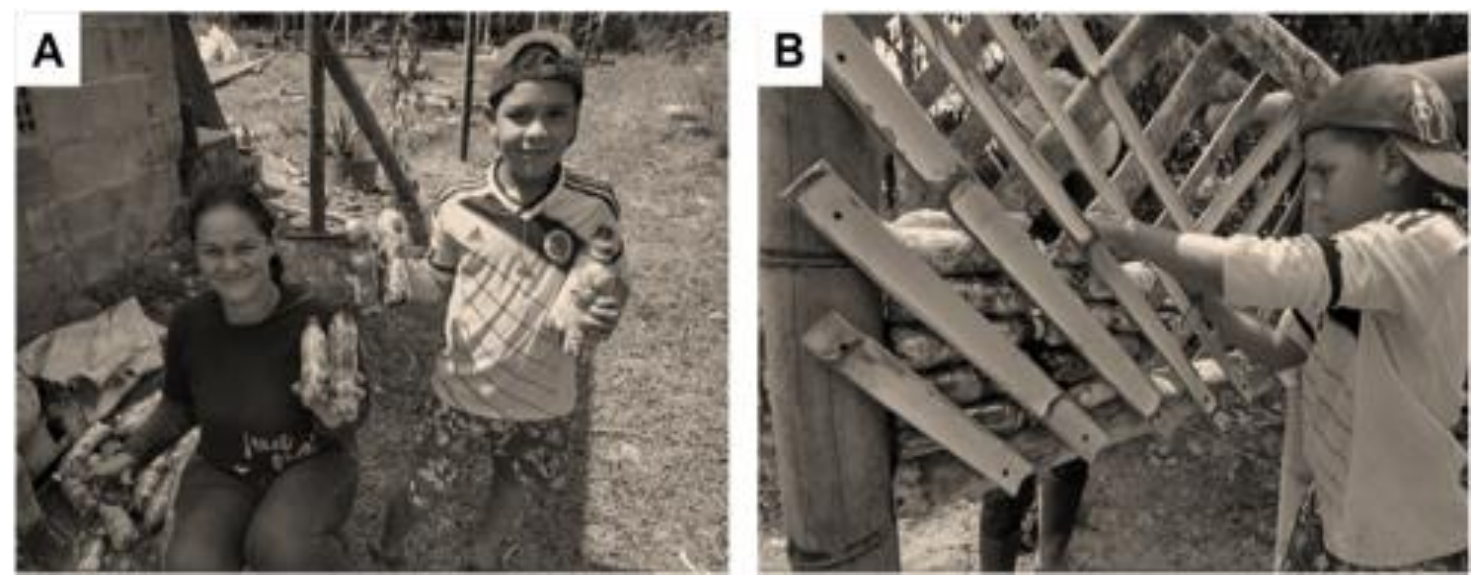

Figura 8. Proceso de sensibilización: A) Integración familiar y B) Instalación de botellas Fuente: elaboración propia.

La cantidad de residuos por botella fue determinada mediante la diferencia de pesos entre botellas con y sin envolturas, donde se estableció un valor por botella vacía de $0.02 \mathrm{~kg}$ y botella llena de $0.12 \mathrm{~kg}$, para un valor aproximado de residuos de $0.1 \mathrm{~kg}$ por botella de $600 \mathrm{ml}$; fuera de lo anterior, se determinó la cantidad de botellas a utilizar por $\mathrm{m}^{2}$ de muro con espesor de $0.12 \mathrm{~m}$, correspondiente al galpón. Para este análisis las botellas utilizadas en su gran mayoría presentaban una longitud de $0.24 \mathrm{~m}$ y diámetro de $0.06 \mathrm{~m}$; a partir de la correlación entre alturas, longitudes y ancho de un $1 \mathrm{~m}^{2}$ de muro con las dimensiones de la botella, se estableció un valor de 139 unidades de botellas PET por $\mathrm{m}^{2}$ de muro. Una vez recolectados los materiales (residuales y no residuales) (Tabla 4) de forma conjunta con la comunidad, se procedió con la designación y realización de actividades para el desarrollo de esta etapa (Figura 9), el cual tuvo una duración aproximada de ocho días. Posterior a la culminación del primer prototipo, dicha actividad fue replicada alrededor de tres veces entre la comunidad de El Prodigio y estos procesos fueron direccionados por líderes comunitarios que asumieron la tarea de darle valor a los residuos como material alternativo aplicado a la construcción sostenible (Figura 10).
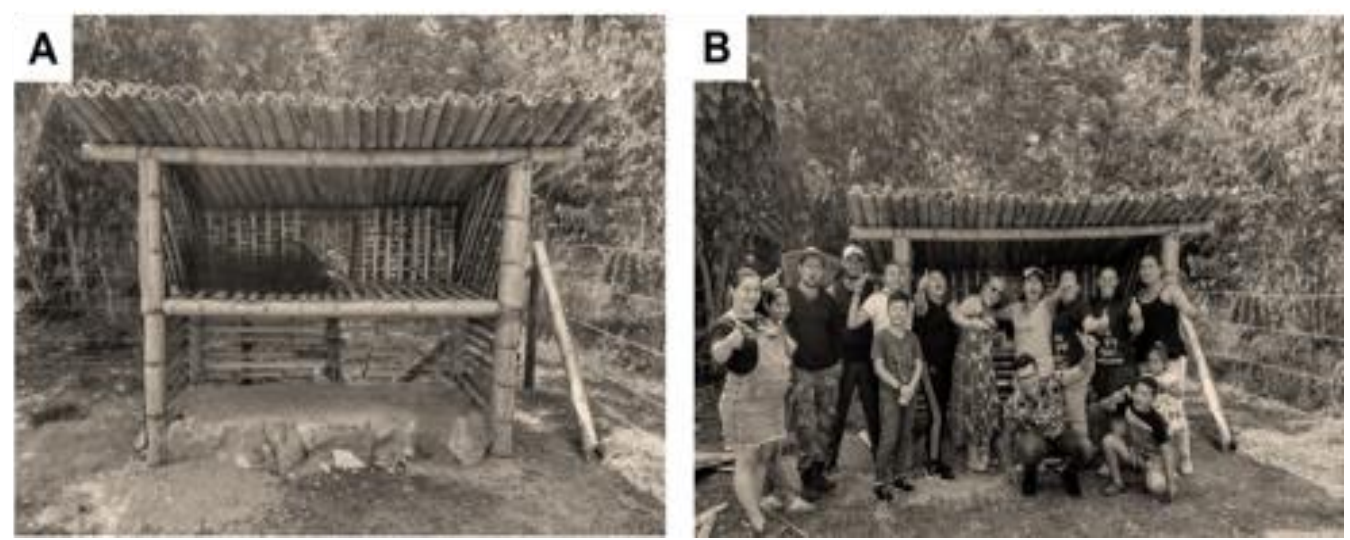

Figura 9. A) Culminación de estructura, B) Retroalimentación de procesos Fuente: elaboración propia. 

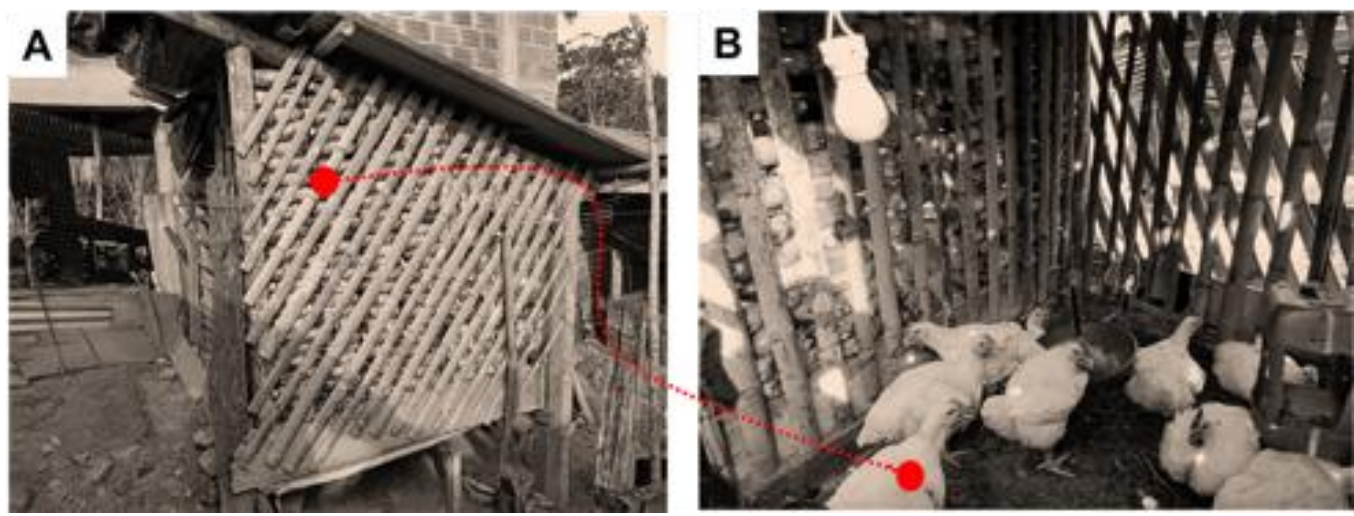

Figura 10. A) Replica de galpones, B) Puesta en funcionamiento de galpón Fuente: elaboración propia.

\section{DISCUSióN}

\subsection{Etapa 5: análisis de durabilidad}

Posterior a la construcción y puesta en funcionamiento de los galpones realizados con materiales residuales y no residuales, se procedió a verificar el comportamiento de los sistemas constructivos frente a los agentes externos mediante procesos de inspección ocular, actividad ejecutada nueve meses después a la puesta en funcionamiento de los galpones. En dicha inspección se pudo determinar que los elementos constructivos correspondientes a la estructura en guadua no presentaban indicios de descomposición, lo único visible correspondía a manchas blanquecinas superficiales debido a la exposición al sol; por otro lado, el revoque de muros (bahareque) no presentaba lesiones relacionadas con humedades, fisuras ni grietas, y la cubierta solo presentó una pequeña filtración, la cual fue solucionada mediante la implementación de barreras físicas impermeables como plástico. El registro de la inspección ocular se muestra en la Figura 11. Además de lo anterior, en algunas guaduas del techo se aprecia la aparición de líquenes, musgo y hongos, debido a la humedad y a la lluvia; es por esto que, para garantizar una mayor durabilidad, se hace necesario en futuras construcciones de proyectos avícolas alternativos, generar procesos de impermeabilización e inmunización en elementos constructivos como la guadua, garantizando así una mayor protección y durabilidad frente a los agentes externos.

Para una futura investigación en comunidades rurales con relación al aprovechamiento de residuos como alternativa de construcción, los autores recomiendan realizar un proceso de indagación con mayor nivel de detalle y desagregación en cuanto a los diferentes materiales a utilizar con su respectiva caracterización físico-mecánica, análisis por elementos finitos de la estructura de soporte de los galpones alternativos y análisis de alternativas constructivas con base en las necesidades de las comunidades en estudio; además, sería pertinente realizar una evaluación ex post a nueve meses de construcción del proyecto como retroalimentación en cuanto a la mejora de los galpones, y al aprovechamiento y uso de los materiales residuales empleados. 

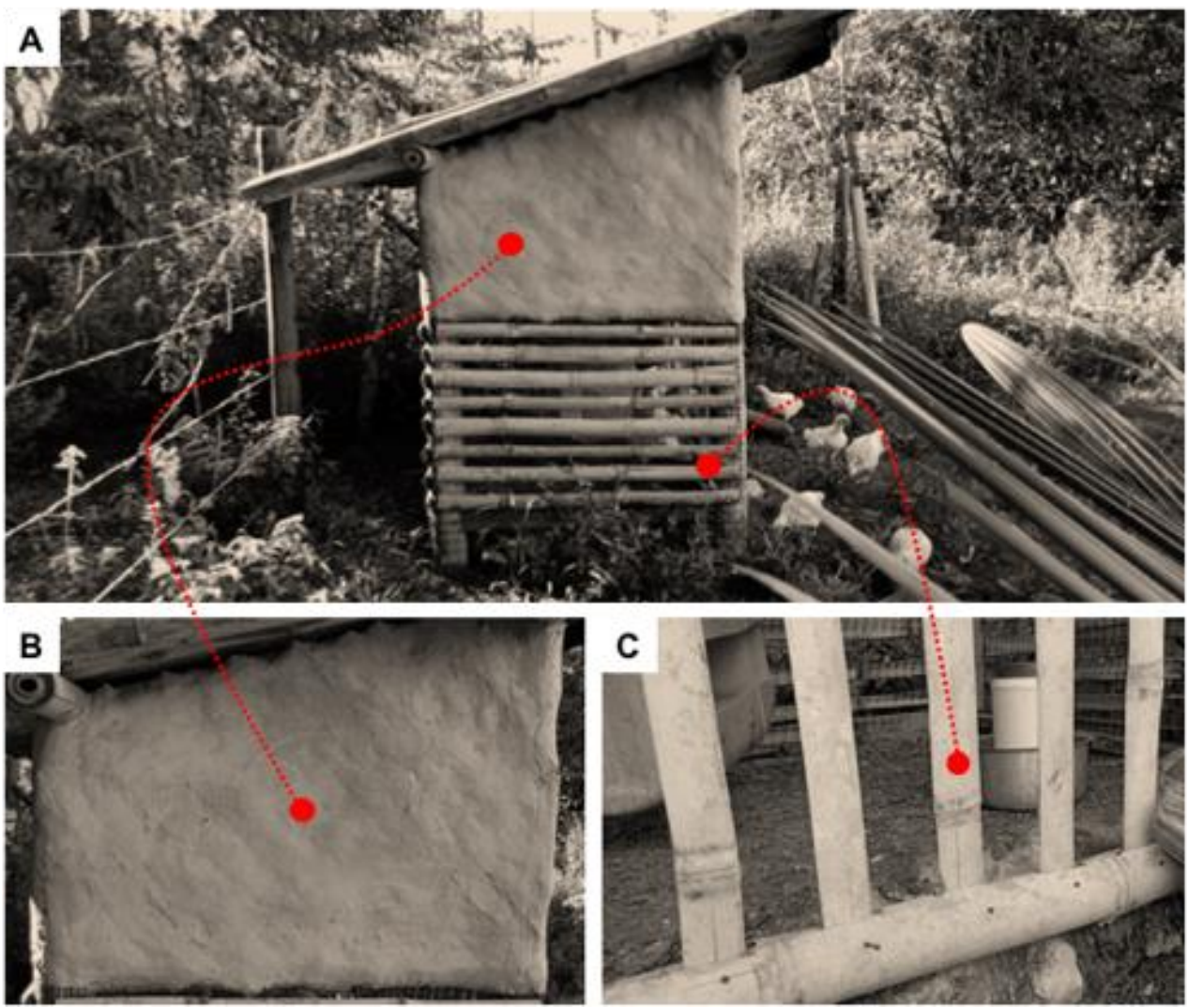

Figura 11. A) Galpón, nueve meses después de su puesta en funcionamiento, B) Revoque sin presencia de lesiones, C) Guadua sin problemas de descomposición. Fuente: elaboración propia.

\section{CONCLUSIONES}

Mediante el reúso de RCD, residuos madereros, restos de alambre, envolturas plásticas y botellas PET en la construcción de proyectos avícolas en zonas rurales, se logran minimizar impactos ambientales, mejorar la economía comunitaria y generar procesos de integración social; lo anterior debido al trabajo cooperativo, a la integración universidad-sociedad y a la aplicación de la construcción sostenible en la realización de galpones. La vinculación de los anteriores procesos enfocados a la sostenibilidad permitió la reutilización de materiales residuales que afectaban las zonas boscosas y urbanas del corregimiento El Prodigio.

En este proyecto se implementaron en total $2143 \mathrm{~kg}$ de materiales para la construcción de galpones como alternativa de sostenibilidad, de los cuales el $87 \%$ correspondía a materiales residuales y $13 \%$, no residuales; del total de materiales no residuales, el $95 \%$ pertenecía a materiales naturales propios de la región como es el caso de la guadua Angustifolia Kunth y $5 \%$ a materiales de la industria de la construcción.

La aplicación de la construcción sostenible permitió en este proyecto la concientización ambiental de comunidades rurales y el aprovechamiento de residuos que eran vistos como una problemática, en una oportunidad económica para la autosostenibilidad y sostenibilidad comunitaria, enfocada a la industria avícola. 


\section{AGRADECIMIENTOS}

Esta investigación no pertenece a ningún proyecto de investigación que hubiera tenido financiación, sin embargo los autores agradecen a la fundación Aurelio Llano y Fraternidad y a la Institución Universitaria Colegio Mayor de Antioquia, que hicieron posible llevar a las zonas rurales de Antioquia los procesos de formación en la Tecnología en Gestión Ambiental, además los autores agradecen enormemente a los estudiantes de la asignatura de construcción sostenible de este programa tecnológico (periodo 01-2020) y en especial a la Líder social Gloria Nancy Fernández Ceballos, por el apoyo brindado en cada uno de los procesos necesarios para logran la concientización ambiental comunitaria y aplicación de la construcción sostenible en el Corregimiento El Prodigio en San Luis Antioquia.

\section{CONFLICTOS DE INTERÉS DE LOS AUTORES}

Los autores no declaran conflictos de interés.

\section{CONTRIBUCIÓN DE LOS AUTORES}

Hernán Darío Cañola: conceptualización, metodología, validación, análisis formal del trabajo, redacción.

Fidel Granda-Ramírez: análisis de datos, desarrollo de componente gráfico y revisión final del manuscrito.

Kelly Leani Quintero-García: análisis cuantitativo, análisis cualitativo, acompañamiento en el desarrollo de galpones por parte de la comunidad de El Prodigio.

\section{REFERENCIAS}

[1] J. C. Escobar Villegas, "La Historia de Antioquia, entre lo real y lo imaginario. Un acercamiento a la versión de las élites intelectuales del siglo XIX", Rev. Univ. EAFIT, vol. 40, no. 134, pp. 51-79, jun. 2012. URL

[2] G. Pérez Ortega; J. W. Branch Bedoya; M. D. Arango Serna, "El sector minero en el nordeste Antioqueño: Una mirada a la Luz de la teoría de las capacidades y los recursos," Rev. Boletín de Ciencias de la Tierra, no. 25, pp. 111-120, dic. 2009. URL

[3] C. Cuartas Calle; J. Arango Muñoz, "Lista de los Mamíferos (Mammalia: Theria) del departamento de Antioquia, Colombia," Biota Colombiana, vol. 4, no. 1, pp. 65-78, jun. 2003. URL

[4] Corantioquia, "Áreas protegidas”, Gobernación de Antioquia, 2017. URL

[5] O. Buitrago; G. Valencia, "El proceso de paz con las Farc y la cuestión rural en Antioquia," Perf. Coyunt. Económica, no. 22, pp. 113-140, dic. 2013. URL

[6] M. Maya Taborda; G. Muñetón Santa; J. Horbath Corredor, "Conflicto armado y la pobreza en Antioquia Colombia," Apunt. Del Cenes, vol. 37, no. 65, 2018. https://doi.org/10.19053/01203053.v37.n65.2018.5460

[7] J. F. Bernal-García; L. Pineda-Cadavid; A. Cardona-Arango, Un camino a la verdad, construcción de memoria colectiva de las víctimas del conflicto armado del municipio San Luis. Las tomas guerrilleras en el corregimiento El Prodigio, Conciudadanía, Medellín, 2018. URL

[8] Cornare, "Referentes ambientales para la Construcción de los Planes de Desarrollo en los Municipios de la Jurisdicción Cornare 2019-2023”, Antioquia, Gobernación de Antioquia, 2019. URL

[9] A. Sáez; G. Urdaneta; A. Joheni, "Manejo de residuos sólidos en América Latina y el Caribe," Rev. Omnia, vol. 20, no. 3, pp. 121-135, sep. 2014. URL

[10] D. Ruíz Valencia; C. López Pérez; E. Cortes; A. Froese, "Nuevas alternativas en la construcción: botellas pet con relleno de tierra," Apunt. Rev. Estud. sobre Patrim. Cult., vol. 25, no. 2, pp. 292-303, jul. 2012. URL 
[11] P. Lalzarliana Paihte; A. C. Lalngaihawma; G. Saini, "Recycled Aggregate filled waste plastic bottles as a replacement of bricks," Mater. Today Proc., vol. 15, no. 3, pp. 663-668, 2019. https://doi.org/10.1016/j.matpr.2019.04.135

[12] A. K. Jassim, "Recycling of Polyethylene Waste to Produce Plastic Cement," Procedia Manuf., vol. 8, pp. 635-642, Oct. 2017. https://doi.org/10.1016/j.promfg.2017.02.081

[13] M. A. Mansur; K. L. Tan; A. E. Naaman, "Strength of bolted moment connections in ferrocement construction," Cem. Concr. Compos., vol. 32, no. 7, pp. 532-543, Aug. 2010. https://doi.org/10.1016/j.cemconcomp.2010.04.001

[14] S. Safinia; A. Alkalbani, "Use of Recycled Plastic Water Bottles in Concrete Blocks," Procedia Eng., vol. 164, pp. 214-221, Jun. 2016. https://doi.org/10.1016/j.proeng.2016.11.612

[15] J. M. L. Reis; E. P. Carneiro, "Evaluation of PET waste aggregates in polymer mortars," Constr. Build. Mater., vol. 27, no. 1, pp. 107-111, Feb. 2012. https://doi.org/10.1016/j.conbuildmat.2011.08.020

[16] I. I. Akinwumi; A. H. Domo-Spiff; A. Salami, "Marine plastic pollution and affordable housing challenge: Shredded waste plastic stabilized soil for producing compressed earth bricks," Case Stud. Constr. Mater., vol. 11, e00241, Dec. 2019. https://doi.org/10.1016/j.cscm.2019.e00241

[17] R. Dachowski; P. Kostrzewa, "The Use of Waste Materials in the Construction Industry," Procedia Eng., vol. 161, pp. 754-758, 2016. https://doi.org/10.1016/j.proeng.2016.08.764

[18] A. M. Wagih; H. Z. El-Karmoty; M. Ebid; S. H. Okba, "Recycled construction and demolition concrete waste as aggregate for structural concrete," HBRC J., vol. 9, no. 3, pp. 193-200, May. 2013. https://doi.org/10.1016/j.hbrcj.2013.08.007

[19] D. Jones; C. Brischke, "2- Wood as bio-based building material," Performance of Bio-based Building., pp. 21-96, 2017. https://doi.org/10.1016/B978-0-08-100982-6.00002-1

[20] D. Altuncu; M. A. Kasapseçkin, "Management and recycling of constructional solid waste in Turkey," Procedia Eng., vol. 21, pp. 1072-1077, 2011. https://doi.org/10.1016/j.proeng.2011.11.2113

[21] R. Manandhar; J. H. Kim; J. T. Kim, "Environmental, social and economic sustainability of bamboo and bamboo-based construction materials in buildings," J. Asian Archit. Build. Eng., vol. 18, no. 2, pp. 49-59, May. 2019. https://doi.org/10.1080/13467581.2019.1595629

[22] S. H. Ghaffar; M. Burman; N. Braimah, "Pathways to circular construction: An integrated management of construction and demolition waste for resource recovery," J. Clean. Prod., vol. 244, pp. 118710, Jan. 2020. https://doi.org/10.1016/j.jclepro.2019.118710

[23] H. D. Cañola; A. Builes-Jaramillo; C. A. Medina; G. E. González-Castañeda, "Bloques de Tierra Comprimida (BTC) con aditivos bituminosos," TecnoLógicas, vol. 21, no. 43, pp. 135-145, sep. 2018. https://doi.org/10.22430/22565337.1061

[24] E. A. Adam; A. R. A. Agib, "Compressed Stabilised Earth Block Manufacture in Sudan," UNESCO. United Nations Educational, Scientific and Cultural Organization, 2001. URL

[25] W. Carazas Aedo; A. Rivero Olmos, Bahareque, guía de construcción parasísmica, CRATerre, Francia, 2002. $\underline{\mathrm{URL}}$

[26] ASTM, "Report of Standard test method for particle - Size analysis of soils ASTM D 422 (Hydrometer)," Report H-130101, west Conshohocken, 2006. URL

[27] P. Perles, Hormigón armado, Buenos Aires, Nobuko, 2006. URL

[28] M. Gutiérrez González; J. I. Bonilla Santos; M. F. Cruz Amado; J. G. Quintero Aranzalez, "Linear expansion and fiber saturation point of guadua angustifolia kunth," Colomb. For., vol. 21, no. 1, pp. 69-80, Jan. 2018. https://doi.org/10.14483/2256201X.11501

[29] Á. Vásquez-Correa; A. M. Ramírez-Arango, Maderas comerciales en el Valle de Aburra, 1st ed. Medellín: Área Metropolitana del Valle de Aburrá, 2005.

[30] ASTM International "ASTM C40/C40M- 20 Standard Test Method for Organic Impurities in Fine Aggregates for Concrete." West Conshohocken, 2014.

[31] F. Awaja; D. Pavel, "Recycling of PET," Eur. Polym. J., vol. 41, no. 7, pp. 1453-1477, Jul. 2005. https://doi.org/10.1016/j.eurpolymj.2005.02.005

[32] P. O. Awoyera; A. Adesina, "Plastic wastes to construction products: Status, limitations and future perspective," Case Stud. Constr. Mater., vol. 12, p. e00330, Jun. 2020, https://doi.org/10.1016/j.cscm.2020.e00330

[33] H. A. Rondón Quintana; F. Reyes Lizcano, "Evaluación de las propiedades mecánicas de una Mezcla densa en caliente modificada con un desecho de PVC," TecnoLógicas, no. 27, pp. 11-31, dic. 2011. https://doi.org/10.22430/22565337.2

[34] S. Shahidan; N. A. Ranle; S. S. Mohd Zuki; F. Sheikh Khalid; A. R. Ridzuan; F. M. Nazri, "Concrete incorporated with optimum percentages of recycled polyethylene terephthalate (PET) bottle fiber," Int. J. Integr. Eng., vol. 10, no. 1, pp. 1-8, Jan. 2018. https://doi.org/10.30880/ijie.2018.10.01.001 
[35] F. Carrasco; P. Pagès; J. Gámez-Pérez; O. O. Santana; M. L. Maspoch, "Processing of poly (lactic acid): Characterization of chemical structure, thermal stability and mechanical properties," Polym. Degrad. Stab., vol. 95, no. 2, pp. 116-125, Feb. 2010. https://doi.org/10.1016/j.polymdegradstab.2009.11.045

[36] Y. H. Wang; W. H. Wang; Z. Zhang; L. Xu; P. Li, "Study of the glass transition temperature and the mechanical properties of PET/modified silica nanocomposite by molecular dynamics simulation," Eur. Polym. J., vol. 75, pp. 36-45, Feb. 2016. https://doi.org/10.1016/j.eurpolymj.2015.11.038 